\title{
Channel Modelling and Performance Analysis of the Near Space based on the DS Signal System
}

\author{
Ke Sun , Xiaomin Hou and Qiang Zhao
}

Equipment Academy, Beijing 101416, China

\begin{abstract}
In order to describe the near space communication channel efficiently and analyse the the reasons that cause quality deterioration of ground-to-air communication link, design the Channel modelling program of the near space and establish the near space channel model based on the DS signal system which considers rain attenuation and multipath fading. Complete the analysis of rainfall impact on signal fading and estimate related parameters under different elevation. Set up the statistical model of multipath fading. Complete the simulation of Ka-band geostationary near space channel performance under different elevation. Draw bit error rate performance simulation curve under different weather conditions. The result of MATLAB simulation shows that the model can reflect the fading characteristic of the near space communication channel. This program improves the signal fading and interference tolerance. The findings can provide a better simulation Platform for the follow-up job according to the correctness and practicability of established model and be of great significance in exploration and research of near space communication.
\end{abstract}

Keywords-near space; rain attenuation; multipath fading; channel model

\section{Introduction}

In recent years, the near space communication system is reflected in the advantages which are the conventional satellite communication system difficult to achieve as a new means of communication. The near space communications platform has many advantages and high military value, such as effective communication, short communication time, small multipath fading, large communication coverage, and strong invulnerability. But the research on near space communications platform focused mainly on aircraft manufacturing, and the achievement of communication technologies is extremely limited, Signal transmission technology is even little suitable for the near space communications[1]. The fundamental reason is the lack of an effective description of the communication channel and construction.

Near space at an altitude of $20 \mathrm{~km}$ to $100 \mathrm{~km}$ in space area, between the satellite platform and aviation platform, which is the must go through place to outer space. At the same time, it is a strategic space for human to develop. As a new research field, many of the technique need to be innovative designed, concluding the measurement \& control and information transmission system. Scientific research institutions at home and abroad had already started the study of tt\&c system in near space because it has a series of advantages: Low cost, rapid deployment, less ground equipment, flexible use, easy to recycle.

There are many patents about spread spectrum in National Patent Office of America. These patents contain design, synchronization, sending, receiving and positioning of PN-code, etc. In the domestic, Tsinghua University, Shanghai Jiaotong University and Beijing University of aeronautics and astronautics and Aerospace science and technology group etc have made a series of innovative research results in the satellite signal capturing and tracking algorithm.

Because of the complexity of communication environment in near space, high-speed movement of communication carrier causes the influence of PN-code phase delay to wireless mobile communication been enhanced. Therefore, the communication system can work effectively and reliably or not, to a large extent depends on the presence of PN-code capture system. At the same time, the tt\&c platforms must possess information security protection, such as anti-interference, resistance to intercept, secret transmission.

Rong-Wei Bao, et al [2] built the channel model to analyze the influence of rain attenuation on the channel at different elevation angles, but did not consider the effects of the ground. Zhong-Min Gan [3] discuss the 
effects of the elevation on the near space communications. Mainly rain attenuation will seriously affect the near-space communication at low elevation angles. But he did not discuss how to improve communication performance near space at moderate elevation and high angles. Yi-Dong $\mathrm{Su}$ [4] built the communication channel model near space Ka-band cell system coverage and analyzed round reflection, rain attenuation on the channel under different elevation angles. But he did not analyze the performance of carrier transmission signal.

With the further development of wireless communication, as one important branch of communication network, near space communication is attracting more and more attention. The characteristics and needs of the near space communication system are combined in this paper. Complete the analysis and simulation of construction of Ka-band geostationary near space channel communications platform. Exactly grasp near space channel characteristics. Complete the construction of the near space communication system transmission model.

\section{The statistical model of near space channel}

According to system components and platform height of the near space, near space communication channel is in line with all the characteristics of Ka-band satellite channel. Therefore, the channel model established is referred to the Ka-band geostationary satellite channel model. Near space platform communication channel is a complex time-varying channel. Not only the path loss and rain attenuation, but also multipath fading and ionosphere are the presence of the transmission signal. Among them, the influence of the ionosphere is negligible at frequencies above $1 \mathrm{GHz}$.

Table 1. The classification and values of Ka-band atmospheric attenuation

\begin{tabular}{ccccc}
\hline $\begin{array}{c}\text { Atmospheric } \\
\text { attenuation of } \\
\text { Ka-band }\end{array}$ & $\begin{array}{c}\text { Atmospheric } \\
\text { absorption } \\
\text { attenuation }\end{array}$ & $\begin{array}{c}\text { Tropospheric } \\
\text { scintillation } \\
\text { decay }\end{array}$ & $\begin{array}{c}\text { Cloud } \\
\text { attenua } \\
\text {-tion }\end{array}$ & $\begin{array}{c}\text { Rain } \\
\text { attenuat } \\
\text {-ion }\end{array}$ \\
\hline $\begin{array}{c}\text { Attenuation } \\
\text { values/dB }\end{array}$ & $\approx 1$ & $<1$ & $\approx 1$ & 42 \\
\hline
\end{tabular}

As can be seen from Table I, it is just considered that atmospheric attenuation is related to the impact of rain attenuation and signal attenuation due to the surrounding environment [5]. Therefore, near space channel model built is mainly composed of two parts: the first is the impact of rain attenuation, the second is the impact of multipath fading.

\subsection{The statistical model of rain attenuation}

Rain attenuation is generated from the radio waves by the absorption of raindrops and the impact of scattering. It is mainly related to geometry of raindrops, rain intensity, rain range, the signal frequency, polarization and so on. Rain attenuation is a major factor affecting the quality of Ka-band communication link.

The effect of rain attenuation is expressed as a timevarying stochastic model [6]:

$$
r_{1}(t)=a(t) \exp (j \phi(t)) s_{1}(t) \quad 0 \leq t \leq T
$$

In the formula, $s_{l}(t)$ and $r_{l}(t)$ is the modulated signal which is respectively used to send and receive. $T$ is the width of modulated symbols. $a(t)$ and $\phi(t)$ is respectively envelope and phase of equivalently low communication channels. Both of them are real random variables. The distributions of probability can be approximated by the Gaussian distribution model [7]:

$$
\begin{aligned}
& p(a)=\frac{1}{\sqrt{2 \pi} \sigma_{1}} \exp \left(-\frac{\left(a-m_{1}\right)^{2}}{2 \sigma_{1}^{2}}\right) \\
& p(\phi)=\frac{1}{\sqrt{2 \pi} \sigma_{2}} \exp \left(-\frac{\left(\phi-m_{2}\right)^{2}}{2 \sigma_{2}^{2}}\right)
\end{aligned}
$$

In the formula, $p(a)$ is probability density function of signal envelope, $p(\phi)$ is probability density function of signal phase, $p(\phi)$ and $\sigma_{2}^{2}$ is respectively variance of envelope and phase. $m_{1}$ and $m_{2}$ is respectively corresponding mean.

Rain attenuation would cause a very big impact on near space communication at low elevation. So this paper mainly considers the situation at low elevation [8].

Table II is composed of relevant parameters of Kaband geostationary near space channel at the elevation angle of $14.2^{\circ}$ under different weather conditions.

Table 2. The channel envelope and phase parameters of the near space

\begin{tabular}{l} 
Weather Mean $m_{1}$ Variance $\sigma_{1}^{2}$ Mean $m_{2}$ Variance $\sigma_{2}{ }^{2}$ \\
\hline Biotite 0.346 \\
Thunderstorm 0.00272 \\
Light rain
\end{tabular}




$$
f_{r}(r)=2 r K \exp \left[-\left(1+r^{2}\right) K\right] I_{0}(2 r K)
$$

In formula, Rice factor is $K=\frac{1}{2 R^{2}}$ which $R^{2}$ is the average scattering power caused by multipath.

If terrestrial user terminals are at different locations, the elevation will be different. With the elevation decreases, the value of factor $K$ in line with the Rice distribution will be also reduced assigned in accordance with Table III [10].

Table 3. Rice distribution statistical properties of different elevation

\begin{tabular}{ccc}
\hline Coverage area & Statistical parameters $/ \mathrm{dB}$ & Ideal values $/ \mathrm{dB}$ \\
\hline High elevation & $10<K<20$ & $K=15$ \\
Moderate elevation & $8<K<10$ & $K=10$ \\
Low elevation & $2<K<6$ & $K=5$
\end{tabular}

\section{Channel modelling program of the near space}

\subsection{The signal system design}

Since the spread spectrum system has more powerful ability of anti-human interference [11], narrow-band interference, anti-multipath interference than the conventional communication system, use the spread spectrum communication technology to improve the signal fading and interference tolerance in the near space communication links in this paper.
The spread spectrum sequences applied to spread spectrum system are commonly $\mathrm{m}$ sequence, gold sequence, $\mathrm{M}$ sequence and so on. Therefore, this paper decides to select the gold code sequence whose length is 1023.

\subsection{The design of modem}

According to the characteristics of the near space monitoring and control communication system and transmission characteristics of the spatial channel[12][14], the choice of digital modulation techniques in this paper is BPSK modulation of high power efficiency design due to sufficient bandwidth of Ka-band.

The block diagram of BPSK modulation and demodulation system is shown in Fig. 1.

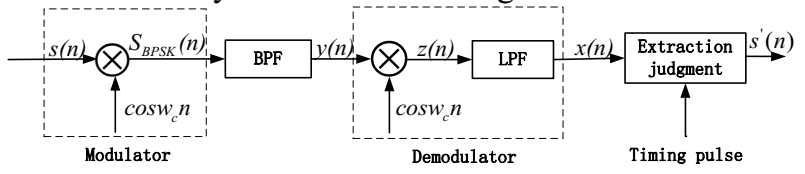

Figure 1. BPSK modulation and demodulation system block diagram.

\subsection{The process design of simulation}

According to the near space channel statistical model, establish the simulation flow diagram as shown in Fig. 2. The binary signal generator generates a continuous stream of binary bits. Pseudo-code sequence generator generates gold code sequence. Multiply them to form a spread spectrum code sequence. After BPSK modulation, multiplicative fading and additive noise pollution of AWGN channel, despread to form the carrier modulated signal and demodulate to recover the original signal. The verdict will be compared with the original symbol as statistical errors. This completes the simulation process design.

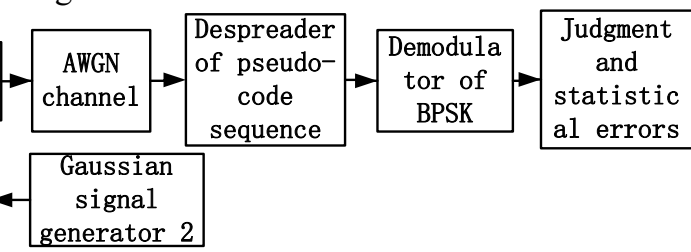

Figure 2. Simulation flow chart of the near space communications system.

The mean and variance generated by Gaussian signal generator 1 is respectively $m_{1}$ and $\sigma_{1}^{2}$. The Gaussian noise process $a(t)$ represents fading process of signal envelope. The Gaussian process $\phi(t)$ of the mean $m_{2}$ and variance $\sigma_{2}^{2}$ is generated by Gaussian signal generator 2 . The phase fading process $\exp (j \phi(t))$ is generated by index generator. It can produce a total attenuation factor $C(t)=a(t) \exp (j f(t))$ that used to simulate the near space channel system with the rice channel and AWGN additive noise.

\section{The performance analysis of near space channel model}

4.1 The performance analysis of the rain attenuation model

Combining with data in Table II, simulate the BER performance of BPSK system in the four types of weather and pure AWGN channel at the elevation of $14.2^{\circ}$. Statistical results of BER are shown in Fig. 3.

Fig. 3 is shown that the bit error rate of the system is changed with SNR curve under different weather conditions. The figure shows that the fading caused by the rain is the least. Compared with pure AWGN channel, the loss of SNR at $10^{-2}$ of BER is about $4 \mathrm{~dB}$ under rain weather conditions. The fading caused by the thunderstorm is relatively enlarged. The loss of SNR at $10^{-2}$ of BER is about $8 \mathrm{~dB}$ under thunderstorm weather conditions. The fading caused by the biotite is the largest. The loss of SNR at $10^{-2}$ of BER is about $10 \mathrm{~dB}$ under biotite weather conditions. The performance of the system cannot be significantly improved when the SNR is greater than $16 \mathrm{~dB}$. 


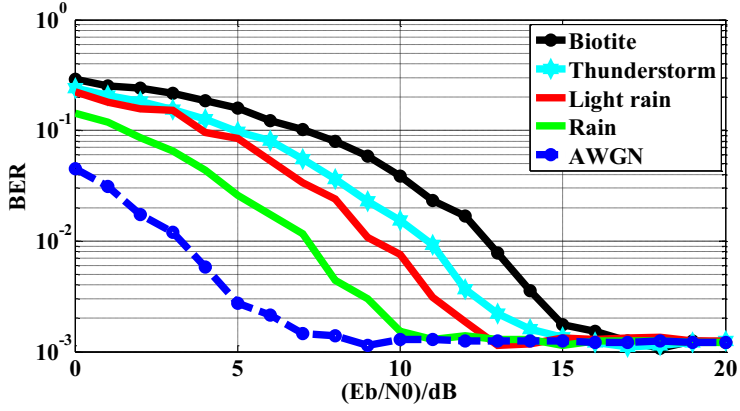

Figure 3. BER simulation of the near space rain attenuation channel.

\subsection{The performance analysis of the multipath fading model}

Combining with data in Table III and Rice channel formula, simulate the BER performance of BPSK system in the three types of elevation and pure AWGN channel. Statistical results of BER are shown in Fig. 4 to 6.

Fig. 4 to 6 are shown that the bit error rate of the system is changed with SNR curve under different weather conditions at the three types of elevation. At different elevation angles, the fading caused by the biotite is the largest. Compared with the pure rain attenuation channel at the elevation of $14.2^{\circ}$, the loss of SNR at $10^{-2}$ of $\mathrm{BER}$ is reduced by $3 \mathrm{~dB}$ at low elevation, $2 \mathrm{~dB}$ at moderate elevation, $1 \mathrm{~dB}$ at high elevation. The fading caused by the thunderstorm and little rain is relatively enlarged. Compared with the pure AWGN channel, the loss of SNR at $10^{-2}$ of BER is about respectively $4 \mathrm{~dB}$ and $3 \mathrm{~dB}$ at low elevation, $5 \mathrm{~dB}$ and $4 \mathrm{~dB}$ at moderate elevation, $6 \mathrm{~dB}$ and $5 \mathrm{~dB}$ at high elevation. The fading caused by the rain is the least. Compared with the pure AWGN channel, the loss of SNR at $10^{-2}$ of BER is about $2 \mathrm{~dB}$ at high elevation. The performance of the system cannot be significantly improved when the SNR is greater than $14 \mathrm{~dB}$.

In contrast with the situation of no fading, the lower the elevation, the lower the bit error rate under all the weather conditions. In the case of direct sequence spread spectrum modulation, increase the signal to noise ratio to be the lowest error rate $10^{-3}$. The simulation results show that near space Ka-band channel quality has been significantly improved with lower elevation.

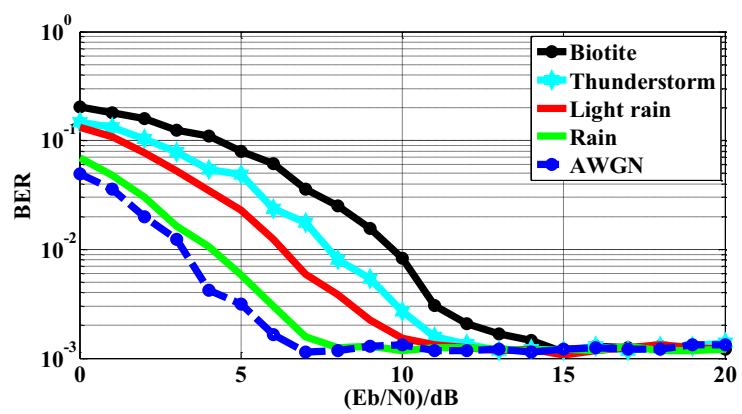

Figure 4. BER simulation of the near space low elevation attenuation channel.

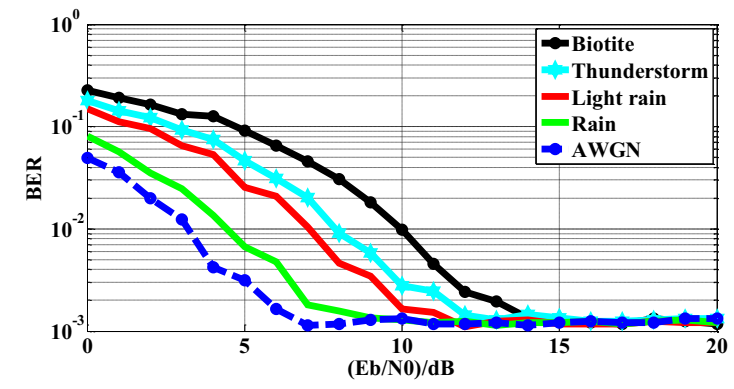

Figure 5. BER simulation of the near space moderate elevation attenuation channel.

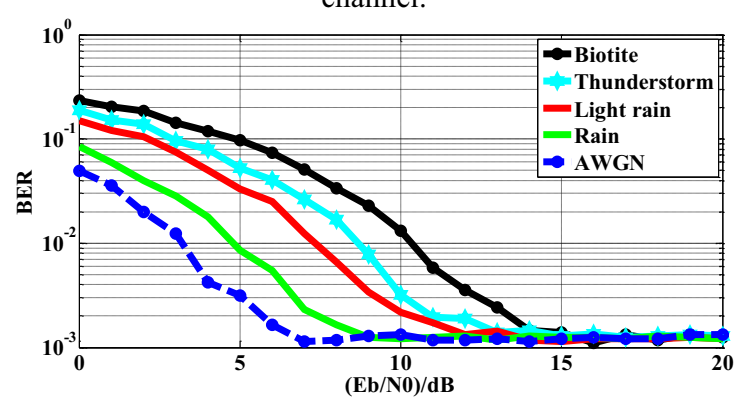

Figure 6. BER simulation of the near space high elevation attenuation channel.

\section{Conclusions}

By analyzing the characteristics of the near space platform communication system and the unique nature of Ka-band channel, analyze the probability distribution of the amplitude and phase of the communication signal on the basis of analyzing the compositions of the near space communication platform, characteristics of the rain attenuation and multipath fading. Show the Ka-band channel model in near space platform. Through further analysis of the physical meaning of the model parameters, estimate the channel model parameters based on the theory of derivation, moreover design of the program of DS signal system channel modelling. At last, simulate and compute the near space channel at different elevations and a variety of weather conditions. At the same time it proves the feasibility and rationality of the model parameters and reflects the significant anti-fading performance of DS signal system. If we can use the appropriate channel coding technology, the transmission performance is expected to have a greater increase. Therefore, this article provides a better environment of simulation for the follow-up job according to the correctness and practicability of established model.

\section{References}

1. M. C. Yang, and Y. C. Jiang, "Fading characteristics of HAPS communication channel in dynamic environment with low elevation angle," Journal of South China University of Technology, vol. 37, no. 1, pp. 27-33, Jan 2009.

2. R. W. Bao, and S. X. Chen, "Near space static channel model rearch," Journal of Air Force Engineering University: Natural Science Edition, vol. 11, no. 6, pp. 22-26, May 2010. 
3. Z. M. Gan, "Near space platform: emergency communication effective means," Digital Communication World, vol. 4, no. 6, pp. 45-49, Sep 2008.

4. Y. D. Su, and S. X. Chen, "Near space Ka-band channel model," Journal of Air Force Engineering University: Natural Science Edition, vol. 12, no. 5, pp. 76-80, Oct 2011.

5. S. Karapantazis, and P. F. Niovi, "Broadband communications via high-Altitude platforms: A survey," IEEE Trans. Surveys\&Tutorials, vol. 7, no. 1, pp. 2-31, Nov 2005.

6. W. Z. Li, C. L. Law, and V. K. Dubey, "Ka-band land mobile satellite channel model incorporating weather effects," IEEE Trans. Communication Letters, vol. 5, no. 5, pp. 194-195, Feb 2001.

7. C. Loo, "Statical models for land mobile and fixed satelite communication at Ka band". IEEE 46th Veh Technol Conf, 1996, Canada, pp. 1023-1027.

8. A. Z. Alejandro, C. R. Jose'Luis, D. P. Jose'Antonio. High-altitude platforms for wireless communications. United Kingdom: John, Wiley \& Sons Ltd, 2008, pp. 123-135.

9. L. C. Jose, A. D. Jos, "Channel model based on semi-Markovian processes. An approach for HAPS systems," Proceedings of the 14th International Conference on Electronics, Communications and Computers, Veracruz, Mexico, 2004, pp. 52-56.

10. J. Han, H. $\mathrm{Xu}$, and $\mathrm{H}$. J. Wu. "HAPS Communication in the low elevation angle the performance analysis," Radio communication technology, vol. 29, no. 6, pp. 51-52, Mar 2003.

11. A. H. Wang, and W. X. Luo. "Ka frequency satellite communication channel building and system performance simulation," Journal of Communication, vol. 22, no. 9, pp. 61-69, July 2001.

12. E. Lutz, D. Cygan, and M. Dippold. "The land mobile satellite communication channel-recording, statistics and channel model," IEEE Trans. on vehicular technology, vol. 40, no. 2, pp. 375-386, July 1991.

13. C. X. Fan. "A kind of new development mobile communication method: research of stratosphere communication". Modern Electronic Technology, vol. 19, pp. 1-3, Jan 2005.

14. C. Lin. "Near space fading channel modeling and its performance analysis," Computer Engineering, vol. 38, no. 6, pp. 80-82, Mar 2012. 